\title{
Structural Complexity in Heterogeneous Catalysis: Cataloging Local Nanostructures
}

\author{
Liudmyla Masliuk, ${ }^{\dagger}$ Marc Heggen, ${ }^{\ddagger}$ Johannes Noack, $^{\dagger, \S}$ Frank Girgsdies, ${ }^{\dagger}$ Annette Trunschke, ${ }^{\dagger}$ \\ Klaus E. Hermann, ${ }^{\dagger}$ Marc Georg Willinger, ${ }^{\dagger, \|}$ Robert Schlögl, ${ }^{\dagger, \perp}$ and Thomas Lunkenbein*, ${ }^{\dagger}{ }^{\dagger}$ \\ ${ }^{\dagger}$ Department of Inorganic Chemistry, Fritz-Haber-Institut der Max-Planck-Gesellschaft, Faradayweg 4-6, 14195 Berlin, Germany \\ ${ }^{\ddagger}$ Ernst-Ruska Centre, Forschungszentrum Jülich GmbH, Wilhelm-Johnen-Straße, 52425 Jülich, Germany \\ ${ }^{\S}$ BasCat-UniCat BASF JointLab, Technical University of Berlin, Hardenbergstraße 36, 10623 Berlin, Germany \\ "Max Planck Institute of Colloid and Interfaces, Am Mühlenberg 1, 14476 Golm, Germany \\ ${ }^{\perp}$ Department of Heterogeneous Reactions, Max Planck Institute for Chemical Energy Conversion, Stiftstraße 34-36, 45470 Mülheim \\ an der Ruhr, Germany
}

\section{Supporting Information}

ABSTRACT: We present an analytical route toward a detailed and quantitative description of individual defects in heterogeneous catalysts. The investigation is based on a high resolution scanning transmission electron microscopy (STEM) study using complex $(\mathrm{Mo}, \mathrm{V}) \mathrm{O}_{x}$ mixed oxide as an example. Tiling the structural regions simplifies the identification of local modifications in the microstructure. Up to 19 different structures were observed that can be listed and classified into different structural motifs, intergrowth, channels, interstitial regions, and inclinations. The observed defects are expressed by the rearrangement of the $\left\{(\mathrm{Mo}) \mathrm{Mo}_{5} \mathrm{O}_{27}\right\}$ building blocks, exhibit different sizes, penetrate the bulk, and can form decoupled surface regions that partially cover the crystallographic bulk. The evaluation of 31 crystals yields an average defect concentration of $3.3 \%$ and indicates the absence of identical particles. We have, for example, observed 54 of these rearranged structures close to the surface of one $(\mathrm{Mo}, \mathrm{V}) \mathrm{O}_{x}$ particle $\left(100 \times 50 \mathrm{~nm}^{2}\right)$. A detailed analysis of the atomic arrangement at the surface of this particle suggests a surface composition of $\left(\mathrm{Mo}_{610} \mathrm{~V}_{230} \mathrm{M}_{70}\right) \mathrm{O}_{x}(\mathrm{M}=\mathrm{Mo}$ and/or $\mathrm{V})$. The resulting catalog of motifs reproduces individual fragments of the real structure of a catalyst and can reveal detailed defect-activity correlations that will contribute to a better understanding of heterogeneous catalysis.

\section{INTRODUCTION}

Heterogeneous catalysis has emerged to a ubiquitous technology, whose products promote our everyday life. Parallel optimizations of catalytic and synthetic protocols of solid catalysts have rendered this technology into the future chemical approach for efficient energy conversion. Those solid catalysts are intrinsically composed of defects in their bulk and surface structure, which can significantly alter the catalytic performance. ${ }^{1}$ Defects, however, are often treated as white spots on the catalytic landscape since they are, due to the lack of translational symmetry, hard to pinpoint. Qualitative and quantitative defect estimation could reflect important defectactivity correlations, which will boost the understanding in heterogeneous catalysis and will simplify a prospective catalyst tailoring.

To bridge this knowledge gap, we focus on a real space, atomic-resolved quantitative defect description using local characterization techniques. Local insight into structural features of limited translational symmetry can be obtained by transmission electron microscopy (TEM) imaging. Recent developments in TEM allow for capturing local fluctuations of the crystallographic bulk structure at the atomic scale and have stimulated research in heterogeneous catalysis. ${ }^{2-4}$ The local analytical strength of TEM has, for instance, provided valuable insights into the surface coverage of $\mathrm{Cu} / \mathrm{ZnO} / \mathrm{Al}_{2} \mathrm{O}_{3}$ catalysts for methanol synthesis ${ }^{5,6}$ or manganese surface enrichment in nanosized $\mathrm{MnWO}_{4}{ }^{7}$

Using aberration corrected scanning transmission electron microscopy (STEM) we explore local structural variations in activated orthorhombic $(\mathrm{Mo}, \mathrm{V}) \mathrm{O}_{x}$, which selectively catalyzes the oxidative dehydrogenation (ODH) of ethane to ethene. ${ }^{8}$ The complex structure of orthorhombic $(\mathrm{Mo}, \mathrm{V}) \mathrm{O}_{x}$ further allows for a broad structural flexibility. ${ }^{9}$ Although details of orthorhombic $(\mathrm{Mo}, \mathrm{V}) \mathrm{O}_{x}$ have already been elucidated by STEM imaging using high angle annular dark field $(\mathrm{HAADF})^{10,11}$ and annular bright field $(\mathrm{ABF})$ detectors, ${ }^{12}$

Received: August 21, 2017

Revised: October 6, 2017

Published: October 10, 2017 


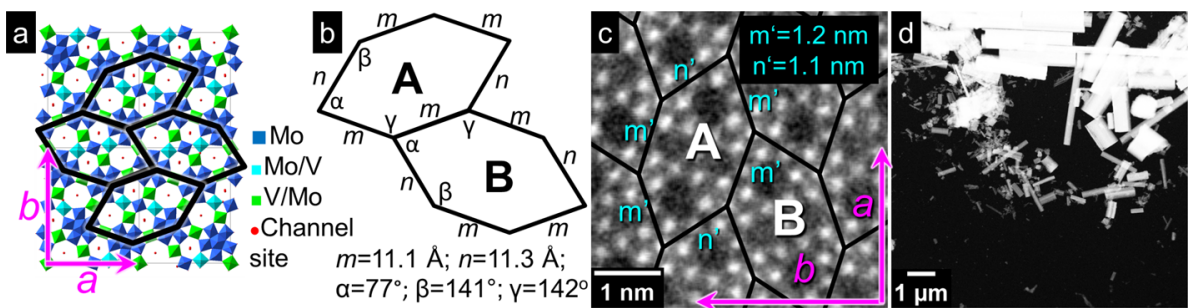

Figure 1. (a) Structural model of the M1 structure viewed along [001]. Black hexagons, standard tiling. Mo/V and V/Mo sites, preferred occupancy of Mo and V, respectively. ${ }^{16}$ (b) Schematic combination of $\mathbf{A}$ and $\mathbf{B}$ tiles $(\mathbf{A B})$ and their lengths $(m, n)$ and angles $(\alpha, \beta)$ as obtained from the ideal crystal structure of the M1 phase. ${ }^{16}$ (c) HAADF-STEM image of the M1 structure viewed along [001] superimposed by the standard tiling. $m^{\prime}$ and $n^{\prime}$, lengths measured directly from the experimental image. (d) Overview HAADF-STEM image of M1 nanorods.

studies on the defect structures of M1 phase mixed oxides are rare. $^{9,10}$ In particular, an in-depth and quantitative defect analysis that describes local atomistic and chemical details of structural parameter variations within this complex catalyst is missing.

In selective oxidation catalysis, a detailed knowledge of individual defects may be important for understanding oxygen activation, electron migration to the surface, and reversible/ irreversible surface enrichments.

Our approach is based on a simple visualization of local structural anomalies using the concept of structural tiling (Figure 1). In brief, the tiling covers the periodic structure with building blocks (tiles) of equal or different shape. ${ }^{13,14}$ Clearly, $2 \mathrm{D}$ tiling does not reflect the complete $3 \mathrm{D}$ structural information. However, a proper tiling model can specifically highlight structural defects that break the perfect periodicity of the otherwise regular, crystalline structure. ${ }^{15}$ Using the concept of tiling our detailed and local investigation identifies new structural motifs that are expressed by new connectivities of $\left\{(\mathrm{Mo}) \mathrm{Mo}_{5} \mathrm{O}_{27}\right\}$ building blocks, intergrowth regions, octagonal channels, interstitial regions, and inclinations. The results are complemented by a statistical analysis and grouped in a catalog of local structures. In addition, alterations of the local composition induced by the structural rearrangements and their general impact on heterogeneous catalysis are also discussed.

\section{METHODS}

Sample Preparation. The orthorhombic $(\mathrm{Mo}, \mathrm{V}) \mathrm{O}_{x}$ mixed oxide (internal ID 20000) was prepared by hydrothermal synthesis. A specially built reactor (Premex Reactor AG, Lengnau, Switzerland) from corrosion resistant Hastelloy C-22 (2.4602) was utilized, which enables precise control of the reaction parameters. A mixture of $9.18 \mathrm{~g}$ of $\left(\mathrm{NH}_{4}\right)_{6} \mathrm{Mo}_{7} \mathrm{O}_{24}$. $4 \mathrm{H}_{2} \mathrm{O}$ (AHM, Merck, $52 \mathrm{mmol} \mathrm{Mo}$ ) dissolved in $230 \mathrm{~g}$ of water (Milli-Q, Merck) and $3.30 \mathrm{~g}$ of $\mathrm{VOSO}_{4}$ (Acros Organics, 12.9 mmol V) dissolved in $30 \mathrm{~g}$ of water was loaded into the hydrothermal reactor at room temperature. Residual air was replaced by nitrogen. Subsequently the vessel was heated to $200{ }^{\circ} \mathrm{C}$ at a rate of $1{ }^{\circ} \mathrm{C} / \mathrm{min}$ and held at this temperature for $17 \mathrm{~h}$. The reaction mixture was stirred during the whole experiment at a rate of $100 \mathrm{rpm}$. After cooling to room temperature, a black solid was isolated by filtration (pore 5 glass frit), washed twice with distilled water, and dried at $80{ }^{\circ} \mathrm{C}$ for $16 \mathrm{~h}$. To remove amorphous components in the hydrothermal product, $1 \mathrm{~g}$ of the powder was washed with $25 \mathrm{~mL}$ of oxalic acid solution ( $0.25 \mathrm{M}$, Acros Organics) at $60{ }^{\circ} \mathrm{C}$ for $30 \mathrm{~min}$ under stirring. The solid was centrifuged $(5000 \mathrm{rpm}, 30 \mathrm{~min})$, washed twice with $25 \mathrm{~mL}$ water, and dried at $80{ }^{\circ} \mathrm{C}$ overnight.
Finally, a thermal treatment was performed in argon atmosphere (flow rate $100 \mathrm{~mL} / \mathrm{min}$ ) at $400{ }^{\circ} \mathrm{C}$ (heating rate $10^{\circ} \mathrm{C} / \mathrm{min}$ ) for $2 \mathrm{~h}$ in a rotary tube furnace (Xerion, Freiberg, Germany).

TEM. Aberration corrected scanning transmission electron microscopy (STEM) was performed on a Jeol JEMARM200CF microscope equipped with CEOS CESCOR and CEOS CETCOR hexapole aberration correctors for probe and image forming lenses, respectively, and a cold field emission gun (CFEG) at $200 \mathrm{kV}$. STEM images were recorded with a JEOL high angle annular dark field (HAADF) detector. Prior to the measurements, the powdered material was drop deposited on a silica coated gold TEM grid. Prior to the HAADF-STEM analysis each particle was oriented along the crystallographic $c$ axis.

XRD. The X-ray diffraction (XRD) measurements were performed in Bragg-Brentano geometry on a Bruker AXS D8 Advance II $\theta / \theta$ diffractometer using Ni filtered $\mathrm{Cu} \mathrm{K} \alpha$ radiation and a position sensitive energy dispersive LynxEye silicon strip detector. The sample powder was filled into the recess of a cupshaped sample holder, with the surface of the powder bed being flush with the sample holder edge (front loading).

XRF. The XRF measurements were performed in a Pioneer S4 X-ray Fluorescence Spectrometer from Bruker. The measurements were performed in vacuum using $100 \mathrm{mg}$ of the sample, fused together with $8.9 \mathrm{~g}$ of di-Li-tetraborate, in a form of the glass disc with $40 \mathrm{~mm}$ diameter. The $\mathrm{K} \alpha$ lines were analyzed by applying the calibration factors derived from the FHI-made appropriate standards.

\section{RESULTS}

Orthorhombic Tiling. In general, orthorhombic $(\mathrm{Mo}, \mathrm{V}) \mathrm{O}_{x}$ mixed oxides crystallize in the so-called M1 structure and are composed of pentagonal $\left\{(\mathrm{M}) \mathrm{Mo}_{5} \mathrm{O}_{27}\right\}$ building blocks that are connected via corner-sharing $\mathrm{MoO}_{6}$ or $\mathrm{VO}_{6}$ octahedra and form hexagonal and heptagonal channels (Figure 1a). ${ }^{17-19}$ In ternary $(\mathrm{Mo}, \mathrm{V}) \mathrm{O}_{x}$ mixed oxides the $\left\{(\mathrm{Mo}) \mathrm{Mo}_{5} \mathrm{O}_{27}\right\}$ units can be differently connected and, thereby, determine the crystal structure. ${ }^{17,18}$ The ideal microstructure of orthorhombic $(\mathrm{Mo}, \mathrm{V}) \mathrm{O}_{x}$ viewed along the crystallographic $c$-axis is shown in Figure 1a. As suggested recently, the ideal orthorhombic M1 structure can be described by combining the central cations of the pentagonal $\left\{(\mathrm{M}) \mathrm{Mo}_{5} \mathrm{O}_{27}\right\}$ building blocks along the cornersharing, neighboring, single octahedral sites (Figure 1). ${ }^{20,21}$

In the M1 unit cell the central Mo cations of the $\left\{(\mathrm{Mo}) \mathrm{Mo}_{5} \mathrm{O}_{27}\right\}$ building blocks are situated in a common plane perpendicular to the crystallographic $c$-axis. ${ }^{16}$ Thus, the Mo vertices of this tiling can be connected torsion free which allows for a direct measurement of the lengths and angles of the 

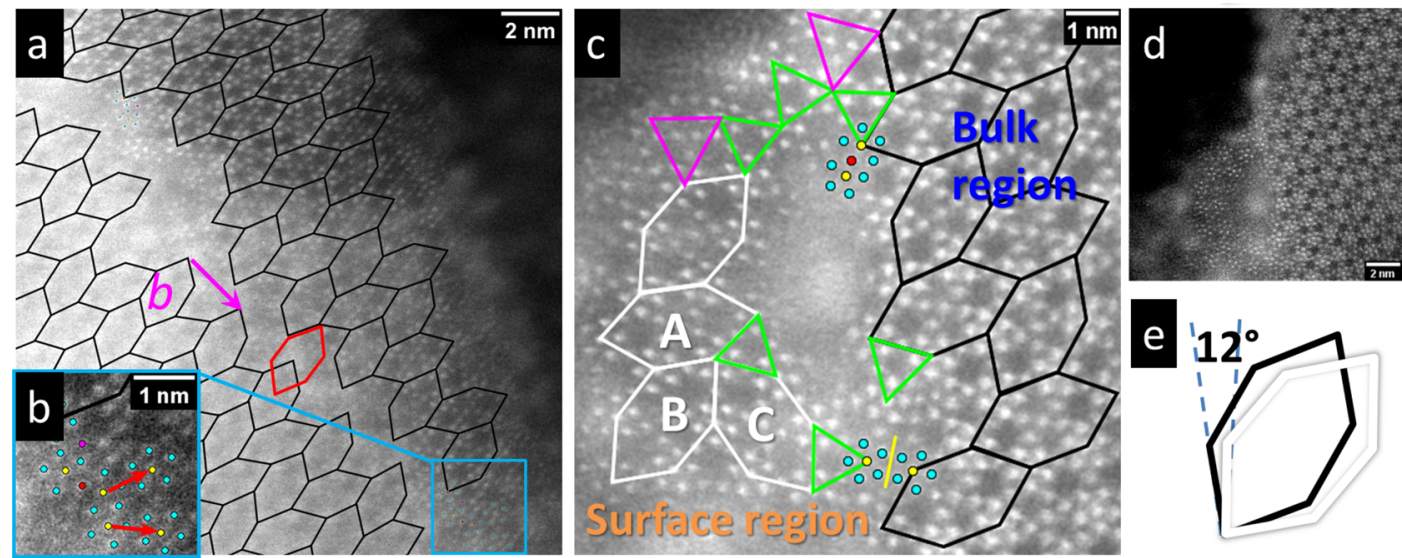

Figure 2. HAADF-STEM images of the M1 structure viewed along [001]. (a) Displacement of separated M1 phases. The red tile highlights the mismatch. (b) Magnified region showing reconnected $\left\{(\mathrm{Mo}) \mathrm{Mo}_{5} \mathrm{O}_{27}\right\}$ units. (c) $\mathrm{M} 1$ tiling applied to the surface region, which is shown in part (d). (e) Schematic representation of the relative orientation of different tiles. Black hexagons, standard tiling; white tiles, rotated standard tiling; yellow and cyan circles, cations of the $\left\{(\mathrm{Mo}) \mathrm{Mo}_{5} \mathrm{O}_{27}\right\}$ units; red and pink circles, unusual positions of cations; red arrow, translation vector; yellow line, mirror plane.

hexagonal tiles (Figure $1 \mathrm{a}-\mathrm{c}$ ). The present tiling is similar to that described for structurally complex, orthorhombic alloys that crystallize in the so-called $\mathrm{T}-\mathrm{Al}-\mathrm{Mn}-\mathrm{Pd}$ phase and exhibit a local 5-fold symmetry with respective tiling angles at the vertices of $72^{\circ}$ and $144^{\circ} . .^{15}$ As opposed to complex metal alloys, the ideal tiling of ternary, orthorhombic $(\mathrm{Mo}, \mathrm{V}) \mathrm{O}_{x}$ mixed oxide derived from the crystal structure ${ }^{16}$ exhibits tiling angles of $77^{\circ}$ and $141^{\circ}$ (Figure $1 \mathrm{~b}$ ). The deviation may result from the influence of the local distortion of different pentagonal blocks that are present in the M1 structure referenced with respect to the central Mo cation in $\left\{(\mathrm{Mo}) \mathrm{Mo}_{5} \mathrm{O}_{27}\right\}$ and depend on the applied synthesis protocol. ${ }^{22,39}$ Each central Mo cation in $\left\{(\mathrm{Mo}) \mathrm{Mo}_{5} \mathrm{O}_{27}\right\}$ is surrounded by five Mo cations where the $\mathrm{Mo}-\mathrm{Mo}_{\text {central }}-\mathrm{Mo}$ angle fluctuates between $68^{\circ}$ and $71^{\circ}$. The corner-sharing octahedra around the $\left\{(\mathrm{Mo}) \mathrm{Mo}_{5} \mathrm{O}_{27}\right\}$ units also form a distorted pentagon that is rotated by $36^{\circ}$ and exhibits $\mathrm{M}-\mathrm{Mo}_{\text {central }}-\mathrm{M}(\mathrm{M}=\mathrm{Mo}, \mathrm{V})$ angles that vary between $70^{\circ}$ and $74^{\circ}$. The corner-sharing octahedra are surrounded by five channels (C), heptagonal and hexagonal, realizing $\mathrm{C}-$ $\mathrm{Mo}_{\text {central }}-\mathrm{C}$ angles from $70^{\circ}$ to $76^{\circ} .{ }^{16}$ The distorted pentagonal channel arrangements are connected by the S1 and S2 sites that act as inversion centers. The $\mathrm{S} 1$ and $\mathrm{S} 2$ cationic sites connect two adjacent heptagonal and hexagonal channels, respectively. In addition, the $\mathrm{S} 2$ site is located in the center of the tiling of the orthorhombic M1 phase. Distortions in the polyhedra of complex $(\mathrm{Mo}, \mathrm{V})$ mixed oxides are not uncommon and depend on the nature of the cations and their oxidation states. ${ }^{12}$ The different oxidation states of the cations confined in the anionic configuration lead to different out-of-center distortions. For instance, fully oxidized molybdenum cations in an octahedral environment exhibit out-of-center displacements that are directed toward faces and edges, whereas $\mathrm{V}^{5+}$ cations are shifted toward the vertices. ${ }^{22}$ Therefore, the complex microstructural arrangement of the orthorhombic M1 phase may be to some extent determined by the atomic displacements of the cations positioned in their individual anionic configuration. In addition, the orthorhombic symmetry of M1 forbids a longrange 5-fold symmetry that is, for instance, observed in quasicrystals.

For the above-mentioned complex metal alloys ${ }^{15}$ the tiles can be transformed into each other by shifting and rotation, while for ternary $(\mathrm{Mo}, \mathrm{V}) \mathrm{O}_{x}$ mixed oxide an additional mirror operation is needed. An $\mathbf{A B}$ combination of tiles is required to describe the unit cell of orthorhombic $(\mathrm{Mo}, \mathrm{V}) \mathrm{O}_{x}$ mixed oxide (Figure 1b). Figure 1c shows a HAADF-STEM image viewed along the $[001]$ zone axis of complex $(\mathrm{Mo}, \mathrm{V}) \mathrm{O}_{x}$ superimposed with combined $\mathbf{A B}$ tiles. Within the error of the measurement the measured angles and distances match the structural parameters obtained from the ideal crystal structure shown in Figure $1 \mathrm{~b}$. In addition, slight imperfections in the orientation of the $(\mathrm{Mo}, \mathrm{V}) \mathrm{O}_{x}$ particle in the electron microscope may result in small variations of the tiling parameters. In this study the tiles were constructed individually for each particle. Further, the labels "ideal tiling" and "standard tiling" refer to the tiling taken from the ideal crystal structure ${ }^{16}$ and from specific $(\mathrm{Mo}, \mathrm{V}) \mathrm{O}_{x}$ particles as obtained from HAADFSTEM imaging, respectively. In agreement with other reports, ${ }^{8,16,21}$ the investigated orthorhombic $(\mathrm{Mo}, \mathrm{V}) \mathrm{O}_{x}$ mixed oxide crystallizes in a rod-like structure (Figure 1d) that grows along the crystallographic $c$-axis. In addition, the measured particle size distribution presented in Figures S1 and S2 confirms an anisotropic particle shape.

XRD analysis (Figure S3) of the investigated M1 phase shows no indications for crystalline impurities or discernible defects in the orthorhombic structure. The observed deviations in the relative reflection intensities from the ideal theoretical values result from preferred crystal orientation effects caused by the crystallite morphology and sample preparation. Local TEM analyses of, altogether, 31 particles reveal the presence of new structural motifs, intergrowth, octagonal channels, interstitial regions, and inclinations. On the basis of local TEM investigations, the average and median defect concentrations, considering all local and visible geometric modifications within orthorhombic $(\mathrm{Mo}, \mathrm{V}) \mathrm{O}_{x}$ mixed oxide, amount to $3.3 \%$ and $1.6 \%$, respectively (Table S1). These defects interrupt the crystallographic bulk structure, form structurally decoupled, extended surface regions, and/or appear individually at the particle surface. XRD is an averaging, integral bulk method, in which the coherently diffracting domains contribute to the mean with the weight of their respective volumes. Hence, the $\mathrm{XRD}$ pattern of the $(\mathrm{Mo}, \mathrm{V}) \mathrm{O}_{x}$ mixed oxide (Figure S3) is dominated by the large domains of the orthorhombic structure and is hardly influenced by the various defects visible in TEM. 


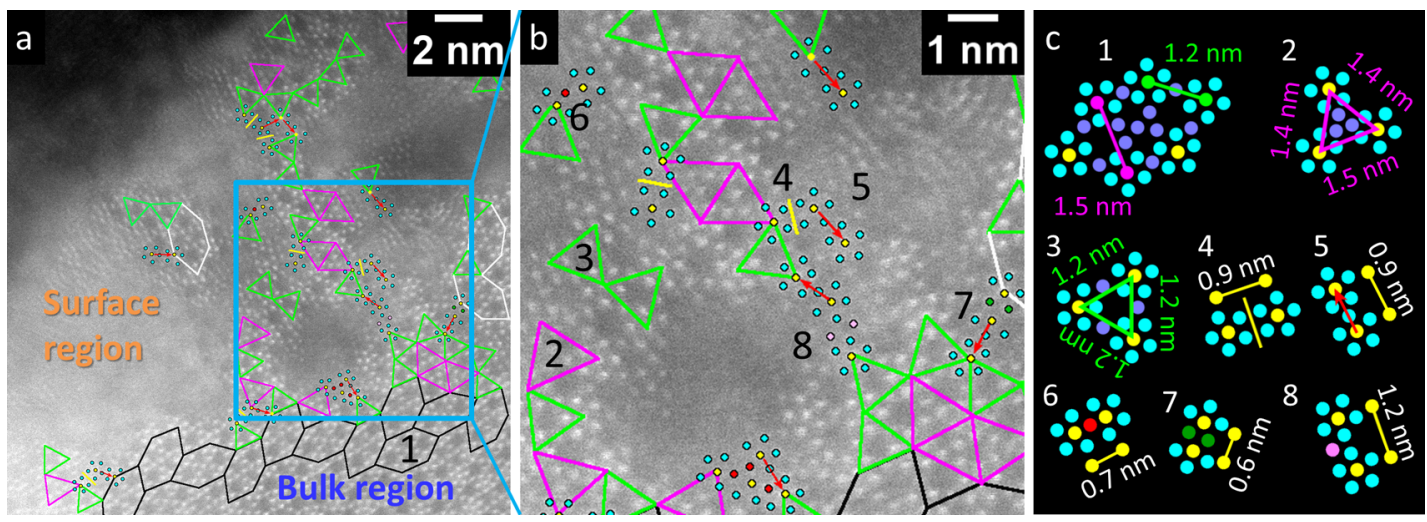

Figure 3. (a) HAADF-STEM image of the surface region and several defect types. (b) Magnified region with all kinds of local motifs. (c) Schematic representation of structural motifs with average distances between central cations of the $\left\{(\mathrm{Mo}) \mathrm{Mo}_{5} \mathrm{O}_{27}\right\}$ units: $(1)$ standard $\mathrm{M} 1$ motif, $(2,3)$ two types of the triangular motifs, (4) mirrored motif, (5) translated motif, (6) shared motif, (7) twinned motif, and (8) rotated motif. Corresponding HAADF-STEM images of each motif are given in Figure S5.

Figure 2 may act as an example of how the local structural analysis benefits from the concept of tiling. The applied tiling indicates structurally decoupled regions. The HAADF-STEM image in Figure 2a appears partially blurred. To identify the reason for blurring, an additional image focusing on the initially blurred part was recorded (Figure S4). The comparison of both images indicates the presence of thickness differences of two structurally separated M1 parts. However, the contrast of Figure $2 \mathrm{a}$ is already high enough to identify the tiling. In addition, Figure 2a highlights a defect layer that discontinues the ideal M1 structure and proceeds along the crystallographic $b$-axis. The displacement vector $(x, y)$ can be expressed by a linear combination of bulk lattice vectors projected onto a $2 \mathrm{D}$ plane with $x$ and $y$ yielding approximately $0.5 a$ and $0.4 b$, respectively, where $a$ and $b$ correspond to the lattice parameters of the ideal crystal structure. The observed displacement in Figure 2a may result from strain relaxation induced by local changes in the connectivities of pentagonal $\left\{(\mathrm{Mo}) \mathrm{Mo}_{5} \mathrm{O}_{27}\right\}$ (Figure 2b) units during synthesis. The tiling, highlighted in Figure 2, also simplifies the identification of surface regions with different connectivities and/or local alterations of the standard M1 structure that would otherwise be overlooked (Figure 2c). These regions partially cover the ideal orthorhombic $(\mathrm{Mo}, \mathrm{V}) \mathrm{O}_{x}$ crystals and occur in $45 \%$ of the investigated particles. Thus, the tiling can act as a measure to directly differentiate the standard bulk structure (black tiling) from local structural modifications observed in the structure (white tiling and triangles in Figure 2c) of the surface region, which is presented in Figure $2 \mathrm{~d}$.

Both standard bulk and surface parts exhibit orthorhombic $(\mathrm{Mo}, \mathrm{V}) \mathrm{O}_{x}$ motifs. The orthorhombic tiles in the surface region, however, display differences that are expressed by a slight rotation (tiles $\mathbf{A}$ and $\mathbf{B}$ ) of $12^{\circ}$ (Figure $2 \mathrm{e}$ ) compared to the standard bulk tiling. Furthermore, tile $\mathbf{C}$ represents a mirrored or rotated image of tile $\mathbf{B}$. These geometric inconsistencies represent local structural rearrangements that can be expressed by changes in the connectivity of the pentagonal $\{(\mathrm{Mo})$ $\left.\mathrm{Mo}_{5} \mathrm{O}_{27}\right\}$ building blocks and induce strain. In the transition region of bulk and surface, structural strain may be relieved by the appearance of triangular motifs. Figure $2 c$ underlines the occurrence of different structural defects that can coexist close to the surface of complex $(\mathrm{Mo}, \mathrm{V}) \mathrm{O}_{x}$ mixed oxide.

Structural Motifs. Figures 2 and $3 a, b$ suggest that the present structural fluctuations originate from different arrange- ments of the pentagonal building blocks where the local connectivity of the $\left\{(\mathrm{Mo}) \mathrm{Mo}_{5} \mathrm{O}_{27}\right\}$ motifs does not reflect the structural arrangement expected for the orthorhombic M1 phase. This modified connectivity of the pentagonal building blocks can be observed in various forms and occurs with different probability (Figure 3c, motifs $1-8$, Figure S5). The new structural motifs that involve two or three neighboring $\left\{(\mathrm{Mo}) \mathrm{Mo}_{5} \mathrm{O}_{27}\right\}$ building blocks can be grouped into two different categories: triangular (Figure 3c, motifs 2, 3) and linear. The linear motifs are distinguished between mirrored, translated, shared, twinned, and rotated motifs (Figure 3c, motifs 4-8), respectively.

In Figure 3c, motif 1 , the standard motif for orthorhombic $(\mathrm{Mo}, \mathrm{V}) \mathrm{O}_{x}$ that corresponds to a single tile is schematically presented. The triangular motifs (Figure 3c, motifs 2,3) can be distinguished by two different connections of the $\{(\mathrm{Mo})$ $\left.\mathrm{Mo}_{5} \mathrm{O}_{27}\right\}$ units and exhibit structural features that have already been observed in the standard M1 tile:

The pentagonal units are connected by two cation sites. The distance between the $\left\{(\mathrm{Mo}) \mathrm{Mo}_{5} \mathrm{O}_{27}\right\}$ building blocks equals the width of the standard tiling (Figure $3 c$, motif 1 ). The triangular arrangement of the $\left\{(\mathrm{Mo}) \mathrm{Mo}_{5} \mathrm{O}_{27}\right\}$ units results in an internal arrangement of cations that can also be found in the trigonal crystal structure (Figure 3c, motif 2).

The pentagonal units are connected by one cation site. The length corresponds to the side of the standard M1 tiling (Figure $3 c$, motif 1 ). This triangular motif exhibits a hexagonal channel and appears in the center of the trigonal unit cell (Figure 3c, motif 3).

The measured interatomic distances inside both triangular motifs match the respective length of the standard tiling of the orthorhombic phase. Therefore, it is expected that the mismatch strain between the triangular motifs and the M1 phase should be small. This might explain the significant enrichment of triangular motifs at the boundary of ordered bulk and surface regions compared to other defects (Figures $2 \mathrm{c}$ and 3 ). Furthermore, changes in the local geometric environment influence the distances and distortions of the pentameric units in the triangular motifs (Figure S6).

A mirrored motif is shown schematically in Figure 3c, motif 4 , and can be described as a $\left\{(\mathrm{Mo})_{2} \mathrm{Mo}_{10} \mathrm{O}_{52}\right\}$ unit in which two octahedra of one $\left\{(\mathrm{Mo}) \mathrm{Mo}_{5} \mathrm{O}_{27}\right\}$ cluster share corners with two octahedra of an adjacent $\left\{(\mathrm{Mo}) \mathrm{Mo}_{5} \mathrm{O}_{27}\right\}$ cluster. Translated motifs are shifted $\left\{(\mathrm{Mo}) \mathrm{Mo}_{5} \mathrm{O}_{27}\right\}$ motifs and form 

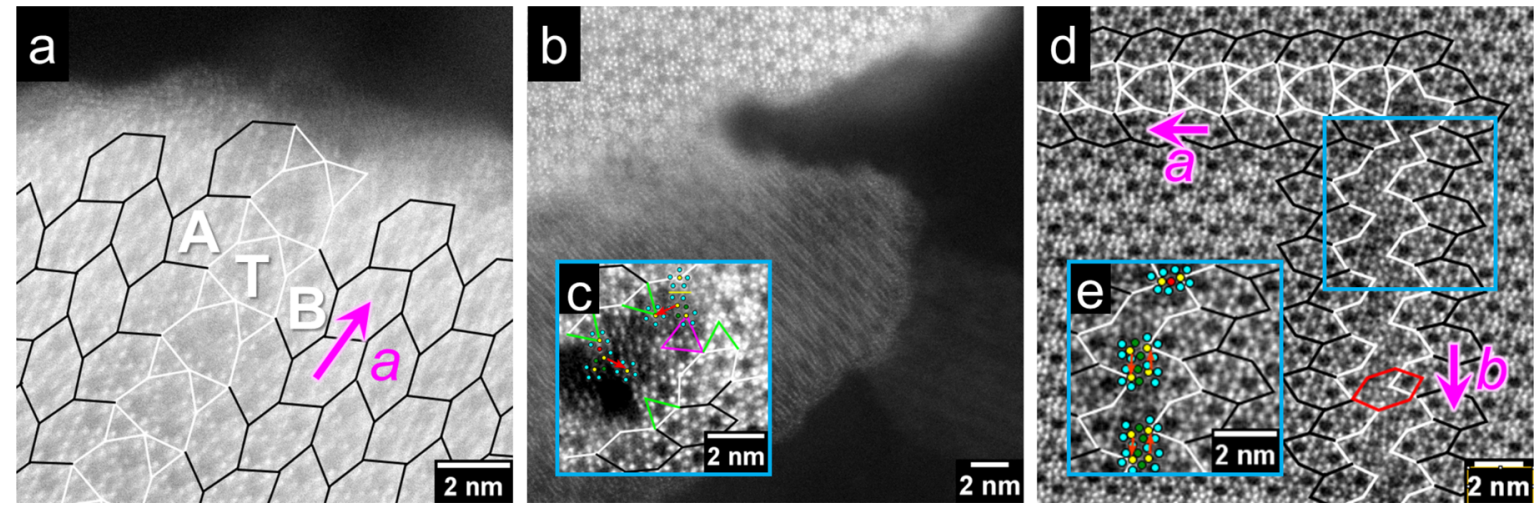

Figure 4. HAADF-STEM images of (a) trigonal, (b, c) aperiodic, and (d, e) zipper-like intergrowth, and their internal structure (c, e). Black hexagons, M1 phase; white arrowhead-shaped tiling, trigonal phase; magenta arrows, unit vectors; green and magenta triangles, trigonal motifs; red arrows, translated motif; yellow line, mirror motif; red circles, shared motifs; green circles, twinned motifs.

corner-shared $\left\{(\mathrm{Mo})_{2} \mathrm{Mo}_{10} \mathrm{O}_{52}\right\}$ units (Figure 3c, motif 5). In shared motifs (Figure $3 \mathrm{c}$, motif 6$)$, two $\left\{(\mathrm{Mo}) \mathrm{Mo}_{5} \mathrm{O}_{27}\right\}$ units are connected sharing one $\mathrm{MoO}_{6}$ octahedron providing edgesharing $\left\{\left(\mathrm{Mo}_{2}\right) \mathrm{Mo}_{9} \mathrm{O}_{48}\right\}$ linkers. The twinned motifs (Figure $3 \mathrm{c}$, motif 7) are similar to the shared motifs but share two Mo cations. The rotated motif is presented in Figure 3c, motif 8 . It is composed of two pentagonal units and one or two added cations $(\mathrm{Mo}, \mathrm{V})$ that are hosted in an anionic configuration. This motif has been observed only rarely.

The distances between the central Mo cations in neighboring $\left\{(\mathrm{Mo}) \mathrm{Mo}_{5} \mathrm{O}_{27}\right\}$ units depend on the local environment (Figure 3 and Figure S6), but as a general trend the distances between the $\left\{(\mathrm{Mo}) \mathrm{Mo}_{5} \mathrm{O}_{27}\right\}$ units decrease in the following order: regular $\left(\sim 1.5 \mathrm{~nm}, \mathrm{Mo}_{\text {central }}-\mathrm{Mo}_{\text {central }}\right.$ distance along the width of the hexagonal tiling; $\sim 1.2 \mathrm{~nm}, \mathrm{Mo}_{\text {central }}-\mathrm{Mo}_{\text {central }}$ distance along the side of the hexagonal tiling $) \geq \operatorname{rotated}(\sim 1.2 \mathrm{~nm}$, $\mathrm{Mo}_{\text {central }}-\mathrm{Mo}_{\text {central }}$ distance $)>$ mirrored/translated $(\sim 0.9 \mathrm{~nm}$, $\mathrm{Mo}_{\text {central }}-\mathrm{Mo}_{\text {central }}$ distance $)>$ shared/twinned $(\sim 0.6 \mathrm{~nm}$, $\mathrm{Mo}_{\text {central }}-\mathrm{Mo}_{\text {central }}$ distance). The influence of the local environment and connectivity on the $\mathrm{Mo}_{\text {central }}-\mathrm{Mo}_{\text {central }}$ distance is illustrated in Figure S6 for different triangular motifs (Figure S6a) and for a tetragonal arrangement of shared motifs (Figure S6b).

Complex orthorhombic $(\mathrm{Mo}, \mathrm{V}) \mathrm{O}_{x}$ is a metastable compound in the phase space of extended oxide structures. The formation of this microstructure requires kinetic control and can be adjusted by the synthesis parameters: small (local) changes in temperature, $\mathrm{pH}$ value, concentration, autogenous pressure, drying procedure, or thermal treatment may cause structural changes at the atomic scale that can be expressed by different connectivities of the pentagonal building blocks. The energy barriers between the different connectivities may be small, offering a variety of different structural motifs. Among the defective structural motifs of the pentameric units, the translated motifs (Figure 3c, motif 5) are observed most frequently suggesting an energetic preference for this structural motif. Rotated motifs (Figure 3c, motif 8 ) are relatively rare indicating a higher formation energy and lower stability. The structurally reconnected $\left\{(\mathrm{Mo}) \mathrm{Mo}_{5} \mathrm{O}_{27}\right\}$ units within the orthorhombic M1 structure might act as local structural defects. Subsequent energy and strain minimization due to the insertion of local defects may lead to structural discontinuities or bulk termination similar to the observations in complex alloys. ${ }^{15}$

Intergrowth Phases. As mentioned in Figure 2a the ideal orthorhombic $(\mathrm{Mo}, \mathrm{V}) \mathrm{O}_{x}$ structure can be discontinued. The intermediate region that separates an ideal M1 crystal can contain any local motifs and tiles. Depending on their geometric arrangement three kinds of different intergrowth phases have been distinguished (Figure 4). The trigonal intergrowth is most common for this kind of ternary mixed oxide (Figure 4a). ${ }^{9-12}$ We have further identified two new intergrowth phases which we label aperiodic (Figure $4 b, c$ ) and zipper-like (Figure 4d,e). The orientation of the crystalline domains before and after the aperiodic intergrowth can differ (Figure $4 \mathrm{~b}$ ). The internal structure of the aperiodic phase can also vary (Figure 4c). The zipper-like intergrowth phase is the rarest and proceeds along the crystallographic $b$-axis (Figures $2 \mathrm{a}$ and $4 d$ ). The measured geometric displacement of two adjacent M1 domains as obtained from Figure $4 \mathrm{~d}$ is $0.5 a$ and $0.3 b$ and corresponds within the error range to the displacement vector derived from Figure 2a. The zipper-like intergrowth contains twinned, shared, and translated motifs (Figure 4e). Different kinds of intergrowth phases can simultaneously occur. Figure $4 \mathrm{~d}$, for instance, highlights the coexistence of zipper-like and trigonal intergrowth phases.

The occurrence of trigonal $(\mathrm{Mo}, \mathrm{V}) \mathrm{O}_{x}$ inclusions in an orthorhombic grain may be attributed to similar synthesis conditions of both phases, which are most commonly obtained by a hydrothermal treatment at different $\mathrm{pH}$ values, ${ }^{23,24}$ and/or may originate from the condensation of individual $(\mathrm{Mo}, \mathrm{V}) \mathrm{O}_{x}$ nanorods. In addition to the structural relaxation mechanism proposed above, which may originate from the differently connected $\left\{(\mathrm{Mo}) \mathrm{Mo}_{5} \mathrm{O}_{27}\right\}$ units, minute local chemical and/or surface potential fluctuations may cause the formation of trigonal phases. Orthorhombic and trigonal phases, however, exhibit different catalytic properties. ${ }^{8}$

For comparison, the crystal structure of the trigonal $(\mathrm{Mo}, \mathrm{V}) \mathrm{O}_{x}$ phase is shown in Figure $5 \mathrm{a}^{23}$ It can be formed by the same $\left\{(\mathrm{Mo}) \mathrm{Mo}_{5} \mathrm{O}_{27}\right\}$ pentagonal building blocks that characterize the orthorhombic structure. ${ }^{25}$ Similar to the M1 phase the central Mo cations of the $\left\{(\mathrm{Mo}) \mathrm{Mo}_{5} \mathrm{O}_{27}\right\}$ units are all located in the same plane perpendicular to the crystallographic $c$-axis. Therefore, these central Mo cations can act as vertices of a trigonal tiling. As opposed to the above-mentioned localized structural motifs the trigonal intergrowth can be interpreted as a planar defect within the orthorhombic phase that lies parallel to the crystallographic $c$-axis and perturbs the orthorhombic structure. The trigonal tiling is depicted as arrowhead-shaped hexagons combined with equilateral triangles (Figure 5). 


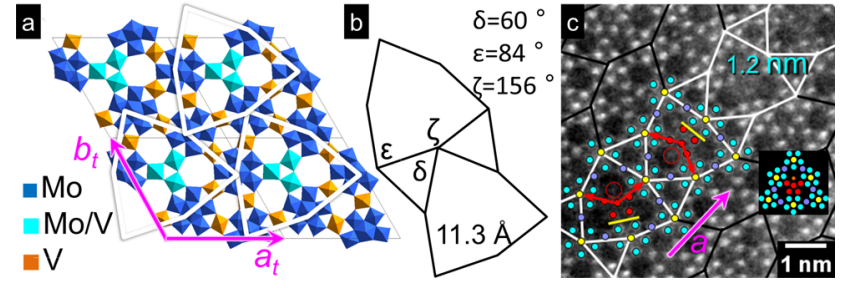

Figure 5. (a) Structural model of the trigonal $(\mathrm{Mo}, \mathrm{V}) \mathrm{O}_{x}$ phase superimposed by tiling. ${ }^{23}$ (b) Schematic representation of the ideal trigonal tiling. ${ }^{23}$ (c) HAADF-STEM image of the trigonal intergrowth viewed along [001], with the inset in part c schematically depicting the internal structure of the pseudotrigonal tiling: black hexagons, M1 phase; white arrowhead-shaped tiling, trigonal phase; red open circles, electron density in the channels; filled red circles, basis of the pentagonal building units; red arrows, translated motifs; yellow lines, mirrored motifs; yellow and cyan circles, cations of the $\{(\mathrm{Mo})$ $\left.\mathrm{Mo}_{5} \mathrm{O}_{27}\right\}$ units; violet circles, corner-sharing cations.

The combination of $2 \mathrm{D}$ tiles provides a comprehensive description of the involved phases. In contrast to the orthorhombic tiling of Figure $1 \mathrm{~b}$, all edge lengths of the proposed trigonal tiles are equal (11.3 $\AA$, Figure $5 \mathrm{~b})$. The HAADF-STEM images in Figures $4 a$ and $5 c$ highlight a trigonal intergrowth in-between an orthorhombic $(\mathrm{Mo}, \mathrm{V}) \mathrm{O}_{x}$ crystal. The measured angles and distances match the geometric parameters obtained from the ideal crystal structure (Figure 5b) within experimental error bars. The trigonal intergrowth separates adjacent orthorhombic phases, but maintains the relative structural relationship. In addition, the periodicity and the direction of the trigonal intergrowth equal the length and direction of the crystallographic a-axis of the orthorhombic phase (Figure 4a).

As shown in Figure 1 the orthorhombic phase is represented by an $\mathbf{A B}$ combination of tiles. The trigonal intergrowth ( $\mathrm{T}$ ) breaks this tiling sequence and results in an ATB arrangement (Figure $4 \mathrm{a}$ ). The individual $\mathbf{A}$ and $\mathbf{B}$ tiles contain boundary edges of two different lengths, 11.1 and $11.3 \AA$, while the trigonal intergrowth (edge length: $11.3 \AA$ ) connects only with the shorter edge of the $\mathbf{A} / \mathbf{B}$ tile (11.1 $\AA$ ). Due to this difference in edge length of $\mathbf{A} / \mathbf{B}$ and $\mathbf{T}$ tiles, the ATB arrangement of tiles is slightly strained. In addition, an ATTB tiling can also exist (Figure S7). Other arrangements that include the intergrowth, such as ATA or BTB, or a connection between sides of equal distance have not been observed.

High resolution HAADF-STEM imaging shows that the trigonal intergrowth (Figure 5c and Figure S8) does not always reflect the ideal trigonal structure (Figure 5a). For example, instead of exhibiting a central V containing triangle (Figure 5a), the $(\mathrm{Mo}, \mathrm{V}) \mathrm{O}_{x}$ mixed oxide can be composed of a pseudopentagonal central unit that is built of four Mo or $\mathrm{V}$ cations with an additional column of lower contrast located in octagonal channels (Figure 5c). A quadrilateral arrangement of cations in the center of the trigonal intergrowth can also appear (Figure S8). Similar to a previous report ${ }^{10}$ we consider this intergrowth as pseudotrigonal. The pseudotrigonal structure in the present $(\mathrm{Mo}, \mathrm{V}) \mathrm{O}_{x}$ mixed oxide with its central pentagonal unit usually exhibits two translated motifs and one mirror motif per trigonal tile (Figure $5 \mathrm{c}$ ). Compared with the ideal $\left\{(\mathrm{Mo}) \mathrm{Mo}_{5} \mathrm{O}_{27}\right\}$ unit the pseudopentagonal center of the pseudotrigonal intergrowth is highly distorted with $\mathrm{M}-$ $\mathrm{M}_{\text {central }}-\mathrm{M}$ angles between $62^{\circ}$ and $81^{\circ}$ (with $\mathrm{M}$ denoting metal cations). In addition, the HAADF-STEM image in Figure $5 \mathrm{c}$ shows a rare case, in which neighboring units of an otherwise regular ordered pseudotrigonal intergrowth are rotated by $115^{\circ}$. Furthermore, the pseudotrigonal intergrowth proceeds through the whole bulk crystal and can also be found at the surface of an orthorhombic $(\mathrm{Mo}, \mathrm{V}) \mathrm{O}_{x}$ mixed oxide (Figure $\left.4 \mathrm{a}\right)$. Similar to previous reports, ${ }^{9,10}$ crystalline trigonal phases at the edges of orthorhombic $(\mathrm{Mo}, \mathrm{V}) \mathrm{O}_{x}$ mixed oxide have also been observed (Figure S9).

Interstitial Regions. The pseudotrigonal intergrowth is occasionally interrupted by local structural modifications. These represent interstitial regions and cause a shift and/or a change of the direction of the intergrowth as highlighted by the arrowhead-shaped trigonal tiling in Figure 6 and Figures S7 and S10-S14. Three different cases were observed as to how adjacent pseudotrigonal intergrowths point at each other before and after the structural intersections (Figure 6) and are schematically summarized in Figure S10: back to back (type-1, Figure 6a), tip to tip (type-2, Figure 6b), and back to tip (type3, Figure 6c).

Interstitial regions with different shapes were found in each pseudotrigonal intergrowth and can appear in both bulk and surface regions (Figure 6 and Figures S10-S14). In Figure 6 the most frequent interstitial regions are highlighted. The interstitial region in Figure 6a and Figure S11 occurs most often and corresponds to a tile of the orthorhombic phase flipped along the long edge of the orthorhombic tile. It is further referred to as a pristine interstitial region. This induces a 2 -fold rotation between tiles leading to the type-1 geometry. An interstitial region that induces a 4-fold rotation between trigonal tiles is shown in Figure $6 \mathrm{~b}$ and Figure S12 and causes
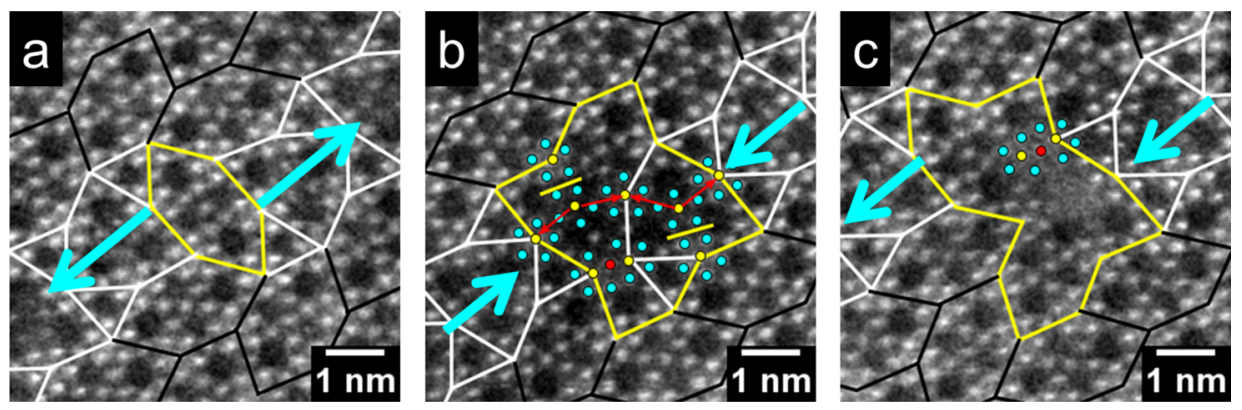

Figure 6. HAADF-STEM images of the most frequent interstitial regions with cyan arrows indicating direction of the pseudotrigonal intergrowth: (a) type-1; (b) type-2; (c) type-3. Yellow polygons, interstitial regions; white tiling, trigonal intergrowth; black tiling, standard M1 structure; yellow and cyan circles, cations of the $\left\{(\mathrm{Mo}) \mathrm{Mo}_{5} \mathrm{O}_{27}\right\}$ pentagonal units; yellow line, mirrored motifs; red arrows, translated motifs; red circles, shared motifs. 

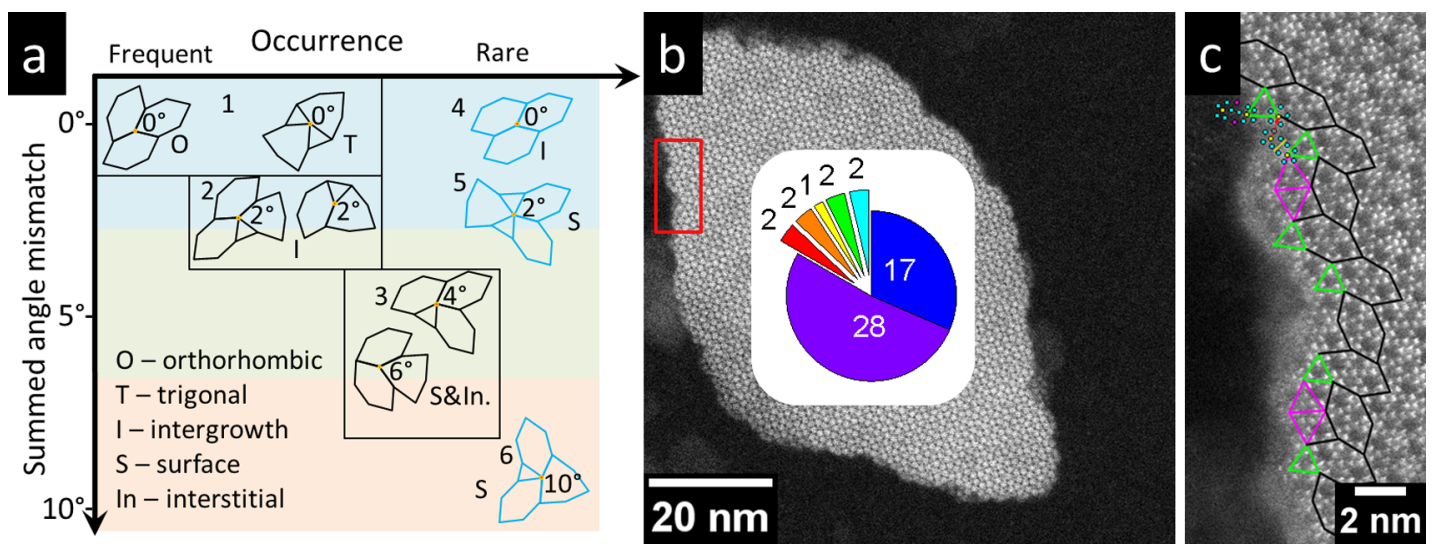

Figure 7. (a) Summary of observed combinations of tiles listed by their frequency of occurrence and mismatch of summed corner angles of joining tiles compared to $360^{\circ}$ : orange circles, corner angles. Labels refer to their location of occurrence. The mismatch calculation is presented and explained in Table S2. (b) HAADF-STEM image of one orthorhombic $(\mathrm{Mo}, \mathrm{V}) \mathrm{O}_{x}$ particle $\left(100 \times 50 \mathrm{~nm}^{2}\right)$ viewed along [001]. The inset in part b highlights the amount of different motifs that occur at the surface of this $(\mathrm{Mo}, \mathrm{V}) \mathrm{O}_{x}$ particle: red, shared; orange, translated; yellow, rotated; green, mirrored; blue, triangular (Figure 3b, motif 2); violet, triangular (Figure 3b, motif 3); cyan, additional cation position (M). (c) Higher magnified HAADF-STEM image of the surface region highlighted in red of the micrograph presented in part $b$.

the type- 2 geometry of the pseudotrigonal intergrowth. More complex interstitial regions with a type- 3 arrangement of trigonal tiles are shown in Figure 6c and Figure S13.

Note that complex interstitial regions can contain all three types of geometrical arrangements (Figure S14). In general, the shift of the pseudotrigonal intergrowth between the two sides of the local structural modification increases with increasing size of the interstitial region. In addition, any structural motifs and tiles mentioned above (Figures $1 b, 3 c$, and $5 b$ ) can be observed in more complex interstitial regions, which differ from the pristine interstitial region introduced in Figure 6a. As for the pristine interstitial region, only the outer local boundary can vary (Figure 6a and Figure S11); additional changes of the internal structure of more complex interstitial regions can be observed while the outer shape is preserved. For example, the 4 -fold rotational interstitial region presented in Figure $6 \mathrm{~b}$ is composed of four translated motifs, two mirrored motifs, and one shared motif, whereas the similar interstitial region presented in Figure S12 is constructed of two translated motifs, one mirrored motif, one rotated motif, and two shared motifs. Both 4-fold rotational interstitial regions host a trigonal tile. As indicated by their different contrast they may have different height with respect to the surrounding ideal orthorhombic structure. Furthermore, Figure S14 shows that the interstitial regions can be significantly larger and are characterized by a low contrast that indicates a higher degree of distortions. These regions frequently contain orthorhombic tiles with additional cation occupancies in the channels that can now be clearly observed in the HAADF-STEM image (Figure S14). In addition, a structure similar to the interstitial regions was observed, which is only surrounded by ideal bulk M1 (Figure S15). We further refer to this defect as an interstitiallike region, which can contain several motifs.

Combinations of Different Tiles. Figure 7 a schematically displays all observed structural combinations of tiles where experimental evidence is given in Figures 1-6 and Figures S11 and S16. The arrangements are grouped according to their occurrence and their mismatch of summed corner angles of joining tiles compared to $360^{\circ}$. Figure 7 a, 1 corresponds to the regular orthorhombic and trigonal tiles (for comparison see Figures 1 and 4). The combinations of tiles shown in Figure
$7 \mathrm{a}, 2$ represent the pseudotrigonal intergrowth (Figures 4 and 5 ). Both combinations appear with the same probability. Less frequent arrangements are shown in Figure 7a,3. They can be found in the surface region (Figure $2 \mathrm{c}$ and Figure S11) and in interstitial regions (Figure $6 \mathrm{a}$ and Figure S14). The rarest combinations of tiles are shown in Figure 7a,4, a,5, and a, 6 and can be found in aperiodic intergrowths (Figure S16a) and at certain spots in the surface regions (Figure S16b,c), respectively. With the exception of $7 a, 4$ and $7 a, 5$, the occurrence decreases with increasing mismatch of summed corner angles of joining tiles. Although the mismatch angle of the arrangement shown in Figure $7 \mathrm{a}, 4$ is $0^{\circ}$, this combination of tiles is rarely observed due to symmetry constraints of the M1 structure. The rare appearance of the arrangement of tiles displayed in Figure 7a,5 may be attributed to a lack of stabilization. In general, the strain induced by these mismatches is relieved by local inclinations or disinclinations that may induce a buckling of the surface. Inclinations may be present in the pseudotrigonal intergrowth (Figures $4 \mathrm{a}$ and $5 \mathrm{c}$ ) and often cause the central pentagon to appear blurred.

In addition, the amount of extended and local defects that are embedded in an orthorhombic $(\mathrm{Mo}, \mathrm{V}) \mathrm{O}_{x}$ crystal can vary noticeably (Table $\mathrm{S} 1$ ). As an example, Figure $7 \mathrm{~b}$ shows an HAADF-STEM image of the M1 structure, which suggests a defect-free bulk region. However, a detailed analysis reveals the presence of at least 54 local defects of different types (Figure $7 \mathrm{~b}$, inset) close to the surface (Figure $7 \mathrm{~b}, \mathrm{c}$ ).

Catalog and Composition of Local Structures. The observed local structures and extended defects together with their individual compositions are summarized in a defect catalog for orthorhombic $(\mathrm{Mo}, \mathrm{V}) \mathrm{O}_{x}$ mixed oxide (Table 1$)$. At present, 19 different configurations have been distinguished in different M1 crystals. While 16 configurations vary in their structural composition, four of them are structurally identical, but are classified as a result of their appearance in different local environments. In addition, no evidence was found that these defective nanostructures could be formed during the exposure of the sample to the electron beam (Figure S17). These local deviations from the ideal M1 structure affect the local composition. The Mo/V ratio within the different defect structures varies (Table 1). For estimates of the defect 
Table 1. Catalog of Observed Structures and Their Compositions

\begin{tabular}{|c|c|c|c|c|c|c|}
\hline Configuration & Schematics & Location $^{b}$ & $\begin{array}{l}\mathrm{Mo} / \mathrm{V} / \mathrm{M} \text { ratio } \\
\text { crystal }^{b} \\
\left.\text { (solely }^{c}\right)\end{array}$ & $\begin{array}{l}\text { Normalized } \\
\text { V content }^{d}\end{array}$ & $\begin{array}{l}\text { Total } \\
\text { number of } \\
\text { appearance }^{e}\end{array}$ & $\begin{array}{l}\text { Total } \\
\text { area of } \\
\text { extended } \\
\text { defects } \\
/ \mathrm{nm}^{2}\end{array}$ \\
\hline \multicolumn{7}{|l|}{ Local motifs } \\
\hline $\begin{array}{l}\text { Triangular } \\
\text { motif (Figure } \\
3 \mathrm{c}, 2 \text { ) }\end{array}$ & & $\mathrm{S}$ & $\begin{array}{l}5.5 / 0.75 \\
(19.25 / 0.75)\end{array}$ & 0.13 & 400 & \\
\hline $\begin{array}{l}\text { Triangular } \\
\text { motif (Figure } \\
3 \mathrm{c}, 3)^{f}\end{array}$ & & S,I & $4 / 3(18 / 3)$ & 0.75 & 690 & \\
\hline $\begin{array}{l}\text { Mirrored } \\
\text { motif }\end{array}$ & xg:8: & S,T,I,In & n.d. ${ }^{g}(12 / 0)$ & 0 & 210 & \\
\hline $\begin{array}{l}\text { Translated } \\
\text { motif }\end{array}$ & x:응 & S,T,I,In & n.d. $(12 / 0)$ & 0 & 380 & \\
\hline Shared motif & & S, In & n.d. $(11 / 0)$ & 0 & 120 & \\
\hline $\begin{array}{l}\text { Twinned } \\
\text { motif }\end{array}$ & 38 & S, In & n.d. (10/0) & 0 & 20 & \\
\hline Rotated motif & Boikc & $\mathrm{S}$, In & $\begin{array}{l}\text { n.d. } \quad(12 / 0 / 1- \\
2)\end{array}$ & $0-0.17$ & 80 & \\
\hline \multicolumn{7}{|l|}{ Tiling } \\
\hline $\begin{array}{l}\text { Orthorhombic } \\
\text { M1 }\end{array}$ & & $\mathrm{O}$ & $\begin{array}{l}15.75 / 4.75 \\
(40.5 / 6.5)\end{array}$ & 0.27 & 986000 & $\begin{array}{l}\text { Compare } \\
\text { Table S1 }\end{array}$ \\
\hline $\begin{array}{l}\text { Orthorhombic } \\
\text { rotated } \\
\text { relatively to } \\
\text { bulk }\end{array}$ & & $\mathrm{S}$ & $\begin{array}{l}15.75 / 4.75 \\
(40.5 / 6.5)\end{array}$ & 0.27 & 160 & \\
\hline \multirow[t]{2}{*}{$\begin{array}{l}\text { Pseudo- } \\
\text { trigonal }\end{array}$} & & $\mathrm{I}, \mathrm{T}$ & $\begin{array}{l}12 / 3.75 \\
(37.5 / 6.75)\end{array}$ & 0.31 & 370 & \\
\hline & & $\mathrm{I}, \mathrm{T}$ & $4 / 3(18 / 3)$ & 0.75 & 720 & \\
\hline \multicolumn{7}{|c|}{ Combined structures } \\
\hline $\begin{array}{l}\text { Quadrilateral } \\
\text { arrangement }\end{array}$ & & I & $\begin{array}{l}10.5 / 3 / 4 \\
(36 / 6 / 4)\end{array}$ & $0.20-0.67$ & 30 & \\
\hline $\begin{array}{l}\text { Additional } \\
\text { cation }\end{array}$ & & S, In & $\begin{array}{l}15.75 / 4.25 / 1 \\
(40.5 / 6.5 / 1)\end{array}$ & $0.25-0.33$ & 70 & \\
\hline \multicolumn{7}{|l|}{ Intergrowth } \\
\hline $\begin{array}{l}\text { Pseudo- } \\
\text { trigonal }\end{array}$ & 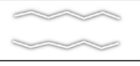 & $\begin{array}{l}\text { along a- } \\
\text { axis }\end{array}$ & n.d. (n.d) & n.d (n.d) & 10 & 1229 \\
\hline Aperiodic & $\begin{array}{l}\text { variable. } \\
\text { highlighted } \\
\text { by a white } \\
\text { outline }\end{array}$ & & n.d (n.d) & n.d (n.d) & 10 & 1861 \\
\hline Zipper-like & & $\begin{array}{l}\text { along b- } \\
\text { axis }\end{array}$ & n.d. (n.d) & n.d (n.d) & 2 & 27 \\
\hline \multicolumn{7}{|c|}{ Interstitial regions } \\
\hline $\begin{array}{l}\text { Pristine } \\
\text { interstitial } \\
\text { region }\end{array}$ & & In & $\begin{array}{l}15.75 / 4.75 \\
(40.5 / 6.5)\end{array}$ & 0.27 & 30 & \\
\hline \multirow{2}{*}{$\begin{array}{l}\text { Four-fold } \\
\text { rotational } \\
\text { interstitial } \\
\text { region } \\
\end{array}$} & & In & $\begin{array}{l}53.63 / 10.50 / 2 \\
(101 / 16.5 / 2)^{i}\end{array}$ & $0.19-0.23$ & \multirow[t]{2}{*}{10} & \\
\hline & & & $\begin{array}{l}53.54 / 14 \\
(99.75 / 19.25)^{\mathrm{j}}\end{array}$ & 0.26 & & \\
\hline $\begin{array}{l}\text { Repetitive } \\
\text { complex } \\
\text { interstitial } \\
\text { region }\end{array}$ & & In & n.d (n.d.) & n.d & 10 & \\
\hline $\begin{array}{l}\text { Complex } \\
\text { interstitial } \\
\text { region }\end{array}$ & $\begin{array}{l}\text { Yellow } \\
\text { polygon } \\
\text { with } \\
\text { variable } \\
\text { shape }\end{array}$ & $\begin{array}{l}\operatorname{In}^{k} \\
\text { bulk }\end{array}$ & n.d (n.d.) & n.d (n.d.) & 10 & 200 \\
\hline
\end{tabular}

${ }^{a} \mathrm{~S}$, surface region; I, intergrowth; T, trigonal phase; In, interstitial region; $\mathrm{O}$, orthorhombic phase. ${ }^{b}$ Nominal content of all cations in the structure, $\mathrm{M}$ corresponds to unidentified sites. Here, Mo, V are not distinguishable. ${ }^{c}$ Nominal content taking the embedding of the local structure in the crystal into account, i.e., shared atoms at edges and vertices. $\mathrm{M}$ corresponds to unidentified sites. Here, Mo, $\mathrm{V}$ are not distinguishable. ${ }^{d} \mathrm{~V}$ content 


\section{Table 1. continued}

normalized to Mo; the interval considers the borders between full occupancies of Mo or V of the unidentified sites. ${ }^{e}$ Counted number of appearance. The rounded values account for the error of counting, and/or the uncertainty when the structure was not clear enough for counting. ${ }^{f}$ There is no difference between the triangular motif and the trigonal tile. Different coloring was only used to separate surface and intergrowth motifs. ${ }^{g}$ Not distinguishable. ${ }^{h}$ Adjacent to both orthorhombic bulk tiles A and B. ${ }^{i}$ Figure 6 b. ${ }^{j}$ Figure S12. ${ }^{k}$ Discontinues the pseudotrigonal intergrowth. ${ }^{l}$ Interstitial-like region, surrounded only by bulk M1 structure and containing at least one motif.

composition, the site occupancy of the different metal centers proposed for orthorhombic and trigonal structures was assumed. ${ }^{16,23}$ The preferred site occupancies in $(\mathrm{Mo}, \mathrm{V})$ based mixed oxides depend on the applied synthetic protocols. $^{16,19,21,26-28}$

For instance, the particle presented in Figure $7 \mathrm{~b}$ is characterized by a defect-free bulk structure, plus a plethora of different structural motifs, which were spotted at the surface of the $(a, b)$-plane of the particle (Figure $7 \mathrm{~b}$ ). With regard to the composition of these local structures (Table 1), we find that the surface has a local composition of $\left(\mathrm{Mo}_{610} \mathrm{~V}_{230} \mathrm{M}_{70}\right) \mathrm{O}_{x}$, where $\mathrm{M}$ denotes metal sites of an uncertain composition $(\mathrm{M}=$ Mo and/or V). This composition can be converted into an $M$ dependent vanadium content normalized to molybdenum, which ranges between 0.3 and 0.5 . For comparison, the overall composition obtained from X-ray fluorescence (XRF) measurement is $\left(\mathrm{MoV}_{0.4}\right) \mathrm{O}_{x}$, while the ideal bulk composition, which is derived from the local configuration (Table 1$)$, is $\left(\mathrm{MoV}_{0.27}\right) \mathrm{O}_{x}$. This result implies compositional differences between bulk and surface structures.

\section{DISCUSSION}

Specific Aspects on Orthorhombic (Mo,V)O $\mathbf{O}_{x}$. The defects observed in orthorhombic $(\mathrm{Mo}, \mathrm{V}) \mathrm{O}_{x}$ mixed oxide resemble those which were found in complex metallic alloys. For instance, the orthorhombic metallic alloy phase T-Al$\mathrm{Pd}-\mathrm{Mn}$ has a local structure with 5-fold symmetry and can be described by a similar hexagon tiling. ${ }^{15,29}$ The structural similarity of the basic pentagonal units in complex metallic alloys promotes a facile intermixing of structural units of different phases, which results in a facile formation of planar defects, dislocations, or phase boundaries. ${ }^{30}$ The resulting low strain connectivities form large and complex defect arrangements that consist of different phases. This behavior has been demonstrated for the intermixing of T-and R-phases or $\varepsilon 6-$ and $\varepsilon 28$-phases in $\mathrm{Al}-\mathrm{Pd}-\mathrm{Mn}$ alloys. ${ }^{15,31,32}$ In addition, overlaps between atomic positions in neighboring pentagonal units reduce the distances between the centers of the units and can be considered as the origin of low strain defects and dislocations. ${ }^{31}$ Similar intermixing and overlapping of structural units of different phases and pentagonal building blocks were observed in orthorhombic $(\mathrm{Mo}, \mathrm{V}) \mathrm{O}_{x}$ mixed oxide in the present study. Thus, the concept of structural intermixing, which causes the formation of different local and extended defects and/or nanostructures that are separated by a low energy barrier, can be transferred from complex alloys to complex mixed oxides with local 5-fold symmetry.

Triangular and linear motifs (mirrored, translated, shared, twinned, and rotated) as well as interstitial and interstitial-like regions described for orthorhombic $(\mathrm{Mo}, \mathrm{V}) \mathrm{O}_{x}$ mixed oxide are cataloged in the present work and are in good agreement with the qualitative observations registered for $(\mathrm{Mo}, \mathrm{V}, \mathrm{Te}, \mathrm{Nb}) \mathrm{O}_{x} \mathrm{M} 1$ and M2 mixed oxides. ${ }^{9,20}$ The shared and twinned motifs are similar to structural defects reported for the pseudohexagonal $\mathrm{M} 2$ phase of multinary $(\mathrm{Mo}, \mathrm{V}, \mathrm{Nb}, \mathrm{Te}) \mathrm{O}_{x}$ mixed oxide. ${ }^{9}$ While in the defective M2 phase the shared and twinned motifs appear with large and small probability, respectively, our study shows that in orthorhombic $(\mathrm{Mo}, \mathrm{V}) \mathrm{O}_{x}$ mixed oxide they occur only with a low probability compared to triangular, mirrored, or translated motifs. The extended pseudotrigonal intergrowth including the central pentagonal motif has already been reported before, but important structural details, for example, the connectivity and orientation of the pentagonal center of the pseudotrigonal intergrowth, were not analyzed. ${ }^{10}$ Compared to the STEM images reported in the literature, ${ }^{10}$ our investigation reveals important local atomistic differences in terms of participating pentagonal units. Previous STEM images show that the pentagonal center of the pseudotrigonal intergrowth is composed of two mirrored and one twinned motif. In our case the observed rotations result in the formation of one mirrored and two twinned motifs. Although this STEM investigation does not focus on synthetic parameters influencing the formation of defects it seems most likely that for this metastable complex mixed oxide slight changes in the synthesis protocol, such as crystallization temperature and time, may affect the defect chemistry.

Details of the interplay of individual structures in these oxides can only be obtained by a three-step local analysis: First, the compositional modifications across individual crystals have to be monitored, and the effects of defects on the chemical bonding have to be understood. Second, the origin and pathways of vanadium surface migration have to be found, and third, the relevant steady state motifs have to be assigned by theory in order to model their electronic structure and to sort local oxygen binding. The first step on this long path has been successfully completed by the present work as far as $(\mathrm{Mo}, \mathrm{V}) \mathrm{O}_{x}$ mixed oxide is concerned.

General Impact on Heterogeneous Catalysis. The surface and bulk structures of model systems and relevant oxidation catalysts are different. ${ }^{33-35}$ Owing to the absence of translational symmetry at the surface, a direct determination of the surface structure is difficult. Surface science has developed techniques that allow the determination of atomic-scale properties of single crystal surfaces usually in ultrahigh vacuum (UHV) with high accuracy. Currently, increasing efforts are devoted to surface structure and reactivity of metal oxide thin films. ${ }^{36}$ Advancements in an atomistic description of the surface structure by thin film studies are limited by the inherent lack of surface-bulk (-support) interactions. Rare insights into the surface-bulk interplay of relevant catalytic systems are obtained by TEM investigations, which reveal the presence of amorphous surface layers ${ }^{37}$ and spectroscopy, which demonstrate a structurally decoupled bulk and surface structure. ${ }^{38}$ In addition, surface instabilities and dynamics complicate the reproduction of their structures. ${ }^{16}$ The activity of a heterogeneous catalyst relies on the balanced interplay of compositional and structural parameters. ${ }^{1,8}$ Atomic differences in the bulk and surface structure of catalysts can be considered as geometric and electronic descriptors, which can discriminate their performance. Defects at the surface, for instance, can be 
considered as high energy centers. These high energy centers influence the surface termination and realize geometric and electronic surface structures, which can (i) regulate the electron-surface supply and (ii) respond differently to the applied chemical potential. ${ }^{16,33}$ Our quantitative study shows that the defect concentration is particle dependent, and, thus, it seems likely that each particle performs differently in a given catalytic reaction. It is worth noting that the activity of selective oxidation catalysts is expected to rely on synergistic surface effects of decoupled and defective surface structures, which can be considered as local nanodomains. ${ }^{39,40}$ The varying defect distribution and intermixing may render a macroscopically homogeneous sample locally heterogeneous. Our study can be considered as a general procedure of how such inter- and intrastructural complexity, far beyond translational-symmetry, can be analyzed. Complementary to the integral information provided by surface spectroscopy, our quantitative real space imaging approach directly pinpoints the positions and distribution of atoms, and their distances and angles. The local structural modifications that are summarized in our defect catalog represent atomistic details that may be responsible for the activity of an oxidation catalyst. To elucidate the influence of the described defects on the catalytic performance, synthetic routines have to be developed to tailor individual local nanostructures at the surface. In addition, theoretical studies that consider the individual and/or a combination of defects are of paramount importance.

\section{CONCLUSIONS}

In summary, the knowledge of the diversity of defects generated by nanostructures combined with a quantitative analysis will establish new defect-activity correlations that will allow an explanation of trends in heterogeneous catalysis in greater detail. The general principle of cataloging local nanostructures shall be further expanded to a quantitative analysis of dynamic surface structures under operation conditions of the catalyst by exploiting quasi in situ TEM techniques.

\section{ASSOCIATED CONTENT}

\section{S Supporting Information}

The Supporting Information is available free of charge on the ACS Publications website at DOI: 10.1021/acs.jpcc.7b08333.

XRD measurement results, statistical information details, extra examples of defects appearance, and the angular deficiency calculations (PDF)

\section{AUTHOR INFORMATION}

\section{Corresponding Author}

*E-mail: lunkenbein@fhi-berlin.mpg.de.

\section{ORCID}

Annette Trunschke: 0000-0003-2869-0181

Marc Georg Willinger: 0000-0002-9996-7953

Thomas Lunkenbein: 0000-0002-8957-4216

Notes

The authors declare no competing financial interest.

\section{ACKNOWLEDGMENTS}

The authors acknowledge Olaf Timpe for XRF measurements. This work was conducted in the framework of the BasCat collaboration between BASF SE, TU Berlin, FHI, and the cluster of excellence "Unified Concepts in Catalysis" (UniCat www.unicat.tu-berlin.de). The authors thank the Max Planck Society for funding.

\section{REFERENCES}

(1) Taylor, H. S. A Theory of the Catalytic Surface. Proc. R. Soc. London, Ser. A 1925, 108, 105-111.

(2) Zhang, Q.-H.; Xiao, D.-D.; Gu, L. Aberration-Corrected Scanning Transmission Electron Microscopy for Complex Transition Metal Oxides. Chin. Phys. B 2016, 25, 066803.

(3) Yang, J. C.; Small, M. W.; Grieshaber, R. V.; Nuzzo, R. G. Recent Developments and Applications of Electron Microscopy to Heterogeneous Catalysis. Chem. Soc. Rev. 2012, 41, 8179-8194.

(4) Vendelbo, S. B.; Elkjær, C. F.; Falsig, H.; Puspitasari, I.; Dona, P.; Mele, L.; Morana, B.; Nelissen, B. J.; van Rijn, R.; Creemer, J. F.; et al. Visualization of Oscillatory Behaviour of Pt Nanoparticles Catalysing CO Oxidation. Nat. Mater. 2014, 13, 884-890.

(5) Lunkenbein, T.; Schumann, J.; Behrens, M.; Schlögl, R.; Willinger, M. G. Formation of a $\mathrm{ZnO}$ Overlayer in Industrial $\mathrm{Cu}$ / $\mathrm{ZnO} / \mathrm{Al}_{2} \mathrm{O}_{3}$ Catalysts Induced by Strong Metal-Support Interactions. Angew. Chem., Int. Ed. 2015, 54, 4544-4548.

(6) Lunkenbein, T.; Girgsdies, F.; Kandemir, T.; Thomas, N.; Behrens, M.; Schlögl, R.; Frei, E. Bridging the Time Gap: A Copper/ Zinc Oxide/Aluminum Oxide Catalyst for Methanol Synthesis Studied under Industrially Relevant Conditions and Time Scales. Angew. Chem., Int. Ed. 2016, 55, 12708-12712.

(7) Li, X.; Lunkenbein, T.; Pfeifer, V.; Jastak, M.; Nielsen, P. K.; Girgsdies, F.; Knop-Gericke, A.; Rosowski, F.; Schlögl, R.; Trunschke, A. Selective Alkane Oxidation by Manganese Oxide: Site Isolation of $\mathrm{MnOx}$ Chains at the Surface of $\mathrm{MnWO}_{4}$ Nanorods. Angew. Chem., Int. Ed. 2016, 55, 4092-4096.

(8) Konya, T.; Katou, T.; Murayama, T.; Ishikawa, S.; Sadakane, M.; Buttrey, D.; Ueda, W. An Orthorhombic $\mathrm{Mo}_{3} \mathrm{VOx}$ Catalyst Most Active for Oxidative Dehydrogenation of Ethane among Related Complex Metal Oxides. Catal. Sci. Technol. 2013, 3, 380-387.

(9) Vogt, T.; Blom, D. A.; Jones, L.; Buttrey, D. J. ADF-STEM Imaging of Nascent Phases and Extended Disorder within the Mo-V$\mathrm{Nb}-\mathrm{Te}-\mathrm{O}$ Catalyst System. Top. Catal. 2016, 59, 1489-1495.

(10) Pyrz, W. D.; Blom, D. A.; Sadakane, M.; Kodato, K.; Ueda, W.; Vogt, T.; Buttrey, D. J. Atomic-Level Imaging of Mo-V-O Complex Oxide Phase Intergrowth, Grain Boundaries, and Defects Using HAADF-STEM. Proc. Natl. Acad. Sci. U. S. A. 2010, 107, 6152-6157.

(11) Pyrz, W. D.; Blom, D. A.; Sadakane, M.; Kodato, K.; Ueda, W.; Vogt, T.; Buttrey, D. J. Atomic-Scale Investigation of Two-Component MoVO Complex Oxide Catalysts Using Aberration-Corrected HighAngle Annular Dark-Field Imaging. Chem. Mater. 2010, 22, 20332040.

(12) Lunkenbein, T.; Girgsdies, F.; Wernbacher, A.; Noack, J.; Auffermann, G.; Yasuhara, A.; Klein-Hoffmann, A.; Ueda, W.; Eichelbaum, M.; Trunschke, A.; et al. Direct Imaging of Octahedral Distortion in a Complex Molybdenum Vanadium Mixed Oxide. Angew. Chem., Int. Ed. 2015, 54, 6828-6831.

(13) Penrose, R. Pentaplexity a Class of Non-Periodic Tilings of the Plane. Mathematical Intelligencer 1979, 2, 32-37.

(14) Hermann, K. Bulk Crystals: Three-Dimensional Lattices. In Crystallography and Surface Structure: An Introduction for Surface Scientists and Nanoscientists; Wiley-VCH Verlag GmbH \& Co. KGaA: Weinheim, 2016; pp 7-89.

(15) Heggen, M.; Houben, L.; Feuerbacher, M. Plastic-Deformation Mechanism in Complex Solids. Nat. Mater. 2010, 9, 332-336.

(16) Trunschke, A.; Noack, J.; Trojanov, S.; Girgsdies, F.; Lunkenbein, T.; Pfeifer, V.; Hävecker, M.; Kube, P.; Sprung, C.; Rosowski, F.; et al. The Impact of the Bulk Structure on Surface Dynamics of Complex Mo-V-Based Oxide Catalysts. ACS Catal. 2017, 7, 3061-3071.

(17) Sadakane, M.; Endo, K.; Kodato, K.; Ishikawa, S.; Murayama, T.; Ueda, W. Assembly of a Pentagonal Polyoxomolybdate Building Block, 
$\left[\mathrm{Mo}_{6} \mathrm{O}_{21}\right]^{6-}$, into Crystalline MoV Oxides. Eur. J. Inorg. Chem. 2013, 2013, 1731-1736

(18) Sadakane, M.; Ueda, W. Building Block Synthesis of Crystalline Mo-V-Based Oxides: Selective Oxidation Catalysts. J. Jpn. Pet. Inst. 2012, 55, 229-235.

(19) Sadakane, M.; Yamagata, K.; Kodato, K.; Endo, K.; Toriumi, K.; Ozawa, Y.; Ozeki, T.; Nagai, T.; Matsui, Y.; Sakaguchi, N.; et al. Synthesis of Orthorhombic Mo-V-Sb Oxide Species by Assembly of Pentagonal $\mathrm{Mo}_{6} \mathrm{O}_{21}$ Polyoxometalate Building Blocks. Angew. Chem., Int. Ed. 2009, 48, 3782-3786.

(20) Blom, D. A.; Vogt, T.; Allard, L. F.; Buttrey, D. J. Observation of Sublattice Disordering of the Catalytic Sites in a Complex Mo-V-NbTe-O Oxidation Catalyst Using High Temperature STEM Imaging. Top. Catal. 2014, 57, 1138-1144.

(21) Ishikawa, S.; Kobayashi, D.; Konya, T.; Ohmura, S.; Murayama, T.; Yasuda, N.; Sadakane, M.; Ueda, W. Redox Treatment of Orthorhombic $\mathrm{Mo}_{29} \mathrm{~V}_{11} \mathrm{O}_{112}$ and Relationships between Crystal Structure, Microporosity and Catalytic Performance for Selective Oxidation of Ethane. J. Phys. Chem. C 2015, 119, 7195-7206.

(22) Ok, K. M.; Halasyamani, P. S.; Casanova, D.; Llunell, M.; Alemany, P.; Alvarez, S. Distortions in Octahedrally Coordinated $\mathrm{d}^{0}$ Transition Metal Oxides: A Continuous Symmetry Measures Approach. Chem. Mater. 2006, 18, 3176-3183.

(23) Sadakane, M.; Watanabe, N.; Katou, T.; Nodasaka, Y.; Ueda, W. Crystalline $\mathrm{Mo}_{3} \mathrm{VO}_{\mathrm{x}}$ Mixed-Metal-Oxide Catalyst with Trigonal Symmetry. Angew. Chem., Int. Ed. 2007, 46, 1493-1496.

(24) Ishikawa, S.; Tashiro, M.; Murayama, T.; Ueda, W. SeedAssisted Synthesis of Crystalline $\mathrm{Mo}_{3} \mathrm{VO}_{\mathrm{x}}$ Oxides and Their Crystal Formation Mechanism. Cryst. Growth Des. 2014, 14, 4553-4561.

(25) Ishikawa, S.; Murayama, T.; Ohmura, S.; Sadakane, M.; Ueda, W. Synthesis of Novel Orthorhombic Mo and V Based Complex Oxides Coordinating Alkylammonium Cation in Its Heptagonal Channel and Their Application as a Catalyst. Chem. Mater. 2013, 25, 2211-2219.

(26) DeSanto, P. j.; Buttrey, D. J.; Grasselli, R. K.; Lugmair, C. G.; Volpe, A. F. j.; Toby, B. H.; Vogt, T. Structural Aspects of the M1 and $\mathrm{M} 2$ Phases in Mo V Nb Te O Propane Ammoxidation Catalysts. Z. Kristallogr. - Cryst. Mater. 2004, 219, 152-165.

(27) DeSanto, P.; Buttrey, D. J.; Grasselli, R. K.; Pyrz, W. D.; Lugmair, C. G.; Volpe, A. F.; Vogt, T.; Toby, B. H. Comparison of MoVTaTeO and MoVNbTeO M1 Crystal Chemistry. Top. Catal. 2006, 38, 31-40.

(28) Murayama, H.; Vitry, D.; Ueda, W.; Fuchs, G.; Anne, M.; Dubois, J. L. Structure Characterization of Orthorhombic Phase in MoVTeNbO Catalyst by Powder X-Ray Diffraction and XANES. Appl. Catal., A 2007, 318, 137-142.

(29) Balanetskyy, S.; Meisterernst, G.; Heggen, M.; Feuerbacher, M. Reinvestigation of the Al-Mn-Pd Alloy System in the Vicinity of the T- and R-Phases. Intermetallics 2008, 16, 71-87.

(30) Feuerbacher, M.; Heggen, M. Elastic Energy of Metadislocations in Complex Metallic Alloys. Acta Mater. 2012, 60, 1703-1711.

(31) Heggen, M.; Feuerbacher, M. Metadislocation Core Structure and Atomic Model for Metadislocation Motion. Acta Mater. 2013, 61, 3851-3857.

(32) Feuerbacher, M.; Heggen, M. Metadislocations. In Dislocations in Solids; Hirth, J. P., Kubin, L., Eds.; Elsevier: Amsterdam, 2010; Vol. 16, pp 109-170.

(33) Schlögl, R. Selective Oxidation: From a Still Immature Technology to the Roots of Catalysis Science. Top. Catal. 2016, 59, $1461-1476$.

(34) Freund, H. J.; Kuhlenbeck, H.; Staemmler, V. Oxide Surfaces. Rep. Prog. Phys. 1996, 59, 283-347.

(35) Sterrer, M.; Freund, H.-J. Properties of Oxide Surfaces. In Surface and Interface Science; Wiley-VCH Verlag GmbH \& Co. KGaA: Weinheim, 2013; pp 229-278.

(36) Kuhlenbeck, H.; Shaikhutdinov, S.; Freund, H.-J. Well-Ordered Transition Metal Oxide Layers in Model Catalysis - a Series of Case Studies. Chem. Rev. 2013, 113, 3986-4034.
(37) Guliants, V. V.; Benziger, J. B.; Sundaresan, S.; Yao, N.; Wachs, I. E. Evolution of the Active Surface of the Vanadyl Pyrophosphate Catalysts. Catal. Lett. 1995, 32, 379-386.

(38) Heine, C.; Hävecker, M.; Sanchez-Sanchez, M.; Trunschke, A.; Schlögl, R.; Eichelbaum, M. Work Function, Band Bending, and Microwave Conductivity Studies on the Selective Alkane Oxidation Catalyst MoVTeNb Oxide (Orthorhombic M1 Phase) under Operation Conditions. J. Phys. Chem. C 2013, 117, 26988-26997.

(39) He, Q.; Woo, J.; Belianinov, A.; Guliants, V. V.; Borisevich, A. Y. Better Catalysts Through Microscopy: Mesoscale M1/M2 Intergrowth in Molybdenum-Vanadium Based Complex Oxide Catalysts For Propane Ammoxidation. ACS Nano 2015, 9, 3470-3478.

(40) Bordes, E. Synergistic Effects In Selective Oxidation Catalysis: Does Phase Cooperation Result In Site Isolation? Top. Catal. 2001, 15, 131-137. 